\title{
Antioxidant activity and total phenolic content of Volvariella volvacea and Schizophyllum commune mycelia cultured in indigenous liquid media
}

\author{
Dulay $\mathbf{R M R}^{1 *}$, Vicente $\mathrm{JJA}^{2}$, Dela Cruz $\mathrm{AG}^{2}$, Gagarin $\mathrm{JM}^{3}$, Fernando $\mathbf{W}^{3}$, \\ Kalaw $\mathbf{S P}^{1}$ and Reyes RG ${ }^{1}$
}

${ }^{1}$ Center for Tropical Mushroom Research and Development, Department of Biological Sciences, College of Arts and Sciences, Central Luzon State University, Science City of Munoz, Nueva Ecija, Philippines

${ }^{2}$ Aliaga National School, Poblacion East II, Aliaga, Nueva Ecija, Philippines

${ }^{3}$ Exequiel R. Lina School, Poblacion Norte, Licab, Nueva Ecija, Philippines

Dulay RMR, Vicente JJA, Dela Cruz AG, Gagarin JM, Fernando W, Kalaw SP, Reyes RG 2016 Antioxidant activity and total phenolic content of Volvariella volvacea and Schizophyllum commune mycelia cultured in indigenous liquid media. Mycosphere 7(2), 131-138, Doi 10.5943/mycosphere/7/2/4

\begin{abstract}
In the current work, we evaluated the different indigenous liquid culture media for mycelial production and antioxidant property based on radical scavenging activity and total phenolic content of Volvariella volvacea and Schizophyllum commune. In $V$. volvacea culture, the maximum mycelial biomass was significantly achieved in coconut water with $12.2 \mathrm{~g}$, while $S$. commune efficiently grew on rice bran broth that produced the highest yield of $12.5 \mathrm{~g}$. Similarly, the highest volume loss of spent was significantly recorded in these media for both mushrooms. Mycelia of $V$. volvacea grown in coconut water and $S$. commune in rice bran broth showed the most potent radical scavenging activity with $21.19 \%$ and $19.45 \%$, respectively. The highest total phenolic content were found in rice bran broth with $23.19 \mathrm{mg}$ AAE/g sample for $V$. volvaceamycelia and in coconut water with $25.52 \mathrm{mg}$ AAE/g sample for $S$. commune mycelia. Therefore, mycelia these edible mushrooms may have potential as natural antioxidants which are affected by the liquid media.
\end{abstract}

Key words $-S$. commune $-V$. volvacea - total phenolic - antioxidant - liquid media

\section{Introduction}

Edible and medicinal mushrooms represent one of the world's greatest untapped resources of nutritious and healthy food. They are rich in protein, carbohydrates, minerals, vitamins, unsaturated fatty acids, phenolic compounds, tocopherols, ascorbic acid and carotenoids (Reis et al.2011). Mushrooms have shown a number of biologically active compounds with therapeutic activities such as, modulation of the immune system, inhibition of tumor growth and inflammation, have hypoglycaemic and antithrombotic activities, lower blood lipid concentrations, prevent high blood pressure and atherosclerosis, and antimicrobial and other activities (Menaga \& Ayyasamy 2012). In addition to this, mushroom fruiting bodies can produce possible resource of optimal quantities of bioactive compounds that exhibit antioxidant properties (Preeti et al. 2012). 
Antioxidants act as the major defense of the body against the damages caused by the free radicals (Jose \& Janardhanan 2000). Chennupati et al. (2012) reported that antioxidants address several diseases like cancer, hepatopathic, nephropathic, retinopathic damage, atherosclerosis, Alzheimer's disease, adult respiratory distress syndrome and diabetes by protecting the cells from oxidative stress; scavenging free radicals, chelating catalytic metals and halting lipid peroxidation chain reactions. The different antioxidants that can derived from foods include phenolic compounds (phenolic acids and flavonoids), carotenoids, tocopherol and ascorbic acids which are classified phytochemicals (Barros et al. 2008). One of the leading techniques in evaluating the antioxidant activity is through the use of 2,2-diphenyl-1-picrylhydrazyl (DPPH), a stable free radical (Chennupati et al. 2012).

Volvariella volvacea is one of the most important and mushrooms for culinary purposes. It is commonly known as paddy straw mushroom or kabuteng saging by Filipinos. Fruiting body of $V$. volvacea is rich in bioactive metabolites and nutritional qualities that contribute not only to its unique and delightful umami taste and aroma but most importantly to its notable functional activities such anti-coagulant, anti-inflammatory, and anti-hypertensive (Eguchi et al. 2015). Schizophyllum commune, on the other hand, is one of the common gill-bearing bracket fungi of world-wide distribution. It can be easily identified by the peculiar structure of its gills which cover hymenium during unfavorable climatic conditions. This mushroom is of great importance in the pharmaceutical and food industries since it produces metabolites which are essential in the production of industrial products (Reyes et al 2009). Schizophyllan is a polysaccharide derived from S. communemycelia that exhibit considerable anticancer activity (Qui \& Liu 2000).

Mushrooms have long been recognized for their unique umami flavour and important biological activities such as antioxidant. Hence, the main objective of the present work was to investigate the antioxidative properties of the two Philippine wild edible mushrooms, $V$. volvacea and $S$. commune, based on their radical scavenging activity and total phenolic content.

\section{Methods}

\section{Mushroom strains and inoculum}

Cultures of $V$. volvacea and $S$. commune were obtained from the Center for Tropical Mushroom Research and Development (CTMRD), Central Luzon State University, Science City of Munoz, Nueva Ecija, Philippines. Mycelia were aseptically revived into sterilized potato dextrose agar (PDA) plates and incubated at $30^{\circ} \mathrm{C}$ for 7 days. These cultures were served as inoculant source.

\section{Mycelial culture in liquid media}

The different indigenous liquid media were used in the evaluation of mycelial production of the two mushroom species. These include coconut water from mature coconut (Cocos nucifera), rice bran decoction (50g of Oryza sativa/L of water), local yellow corn grit decoction $(50 \mathrm{~g}$ of Zea mays/L of water) and potato sucrose broth $(250 \mathrm{~g}$ of Solanum tuberosum/L of water $+10 \mathrm{~g}$ of white table sugar). Liquid media $(100 \mathrm{ml})$ were dispensed into microwavable plastic container and sterilized in an autoclave at $121^{\circ} \mathrm{C}, 15 \mathrm{psi}$ for 30 minutes. There were five replicates per treatment. After cooling, media were inoculated with mycelial discs (prepared using flame sterile 10-mmdiameter cork borer) and incubated at $30^{\circ} \mathrm{C}$ in static condition for 10 days to allow fungal growth. The mycelial mats were harvested and weighed (wet weight basis) and the volume loss of culture spent was measured.

\section{Extraction of active components}

Culture spent and mycelia were homogenized using a food processor and then ethyl acetate $(10 \mathrm{ml})$ was added into each cultured broth to extract antioxidant compounds. The ethyl acetate soluble portion was concentrated under reduced pressure and the concentrates were dissolved in ethanol for antioxidant analysis. 


\section{Radical scavenging activity assay}

The free radical scavenging activity of the samples was estimated using the stable 2,2'diphenyl-1-1picrylhydrazyl (DPPH) radical following the standard method of Shimada et al. (1992) with minor modifications. A $100 \mu \mathrm{l}$ of test sample in ethanol was added with $5 \mu \mathrm{DPPH}$ solution ( $5 \mathrm{mg}$ DPPH powder in $2 \mathrm{ml}$ of ethanol) in 96-well microtitter plates. The mixture was shaken vigorously and left to stand for $30 \mathrm{~min}$ in the dark, and the absorbance was then measured at $517 \mathrm{~nm}$. The inhibition of DPPH free radicals was calculated.

\section{Estimation of total phenolics}

The total phenolic content was estimated using Folin-Ciocalteu method of Slinkard \& Singleton (1977) with modifications. Sample solution $(50 \mu \mathrm{l})$ was mixed $500 \mu \mathrm{l}$ of $10 \%$ FolinCiocalteu reagent (Folin:Methanol, 1:1, v/v). After 2 min, $50 \mu 1$ of $7.5 \%$ saturated was added and kept in the dark for $1 \mathrm{~h}$ before absorbance was taken at $765 \mathrm{~nm}$. A calibration curve was obtained using various concentrations of ascorbic acid. The total phenolic content of the sample was expressed as mg of ascorbic acid equivalents (AAEs) per gram of sample. Data were analyzed using Analysis of Variance (ANOVA) in SAS Statistical Program.

\section{Results and Discussion}

\section{Mycelial production in liquid media}

In mushroom biomass production, culture medium and/or substrate is one of the most important factors for efficient growth. In this present work, the mycelial growth responses of $V$. volvacea and $S$. commune on the different liquid media were investigated and the results are shown in Figure 1. It can be seen that in $V$. volvacea culture, the maximum mycelial biomass was significantly achieved in coconut water having a mean of $12.2 \mathrm{~g}$ at $30^{\circ} \mathrm{C}$ for 12 days. However, rice bran broth supported the most efficient mycelial growth of $S$. commune that produced the highest yield of $12.5 \mathrm{~g}$. These two indigenous liquid media were found statistically comparable with the commercial potato broth (CPB). These results imply that coconut water and rice bran broth could be an alternative media for mycelial biomass production of $V$. volvacea and $S$. commune, respectively. On the other hand, corn grit broth registered the lowest yield of mycelia in both evaluated species. Volume loss of the spent media was also determined after 12 days of incubation and the results are presented in Figure 2. Coconut water (for V. volvacea) and rice bran broth (for S. commune significantly recorded the highest volume loss of the spent whereas con grit broth had the lowest volume loss in both mushrooms. Apparently, liquid media with highest yield of mycelia registered the highest volume loss of the spent, there is, therefore, a positive correlation.

The superiority of coconut water in $V$. volvacea culture and rice bran broth in $S$. commune culture could be attributed to a wide variety of their chemical components that support the efficient mycelial growth. Coconut water is nutritious medium rich sugars, sugar alcohols, lipids, amino acids, nitrogenous compounds, organic acids, enzymes, vitamins (B1, B2, B3, B5, B6, B7 and B9) and minerals vital for physiological functions (Santoso et al. 1996, Yong et al. 2009). It is also contains phytohormones like auxin, various cytokinins, gibberellins (GAs), ethylene, cytokinins, and abscisic acid (ABA) that play crucial roles in regulating plant growth in a wide range of developmental processes (Ma et al. 2008). Rice bran, on the other hand, contains micronutrients such as oryzanol, tocopherols, tocotrienols, phytosterol and dietary fiber like beta-glucan, pectin and gum (Nagendra et al. 2011). It is also contain vitamin B1 and thamine, which are essential for mycelial growth (Silverio et al. 1981).

However, in our previous works, the maximum mycelial yield of Panaeolus cyanescens was achieved in coconut water and rice bran broth (Bustilloset al. 2014) while potato broth produced the most luxuriant mycelial growth of Panaeolus antillarium (Dulay et al. 2015a). Moreover, both Lentinus tigrinus and Lentinus sajor-caju efficiently grew on rice bran broth which significantly recorded the highest yield of mycelia and volume loss (Dulay et al. 2015b). These findings and the results of the present study demonstrated that the efficient mycelial production of these 
basidiomycetes depend on the liquid medium used in submerged cultivation. Submerged cultivation is a standard technique of mycelial biomass production of mushrooms with high nutritional value and functional bioactivity. So, the bioactivities, in particular, antioxidant property of submerged culture of the two evaluated mushrooms was also carried out in this study.

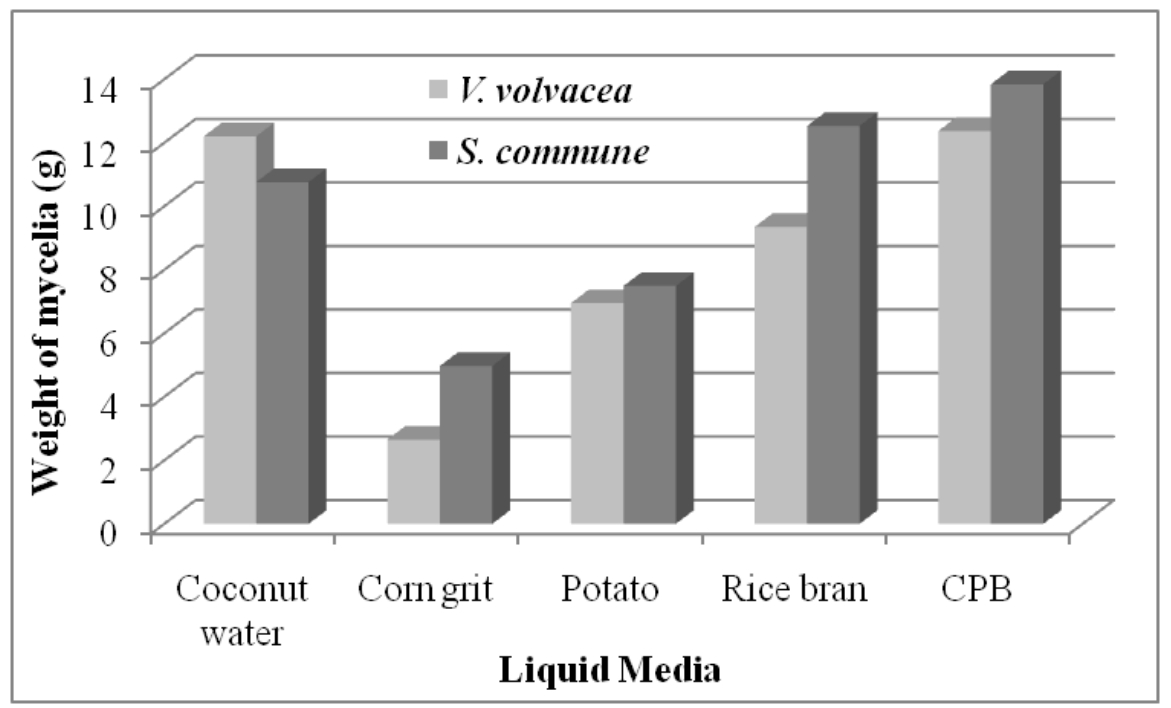

Fig. 1- Yield of mycelial biomass of $V$. volvacea and $S$. commune in different liquid media after 12 days of incubation. CPB; commercial potato broth.

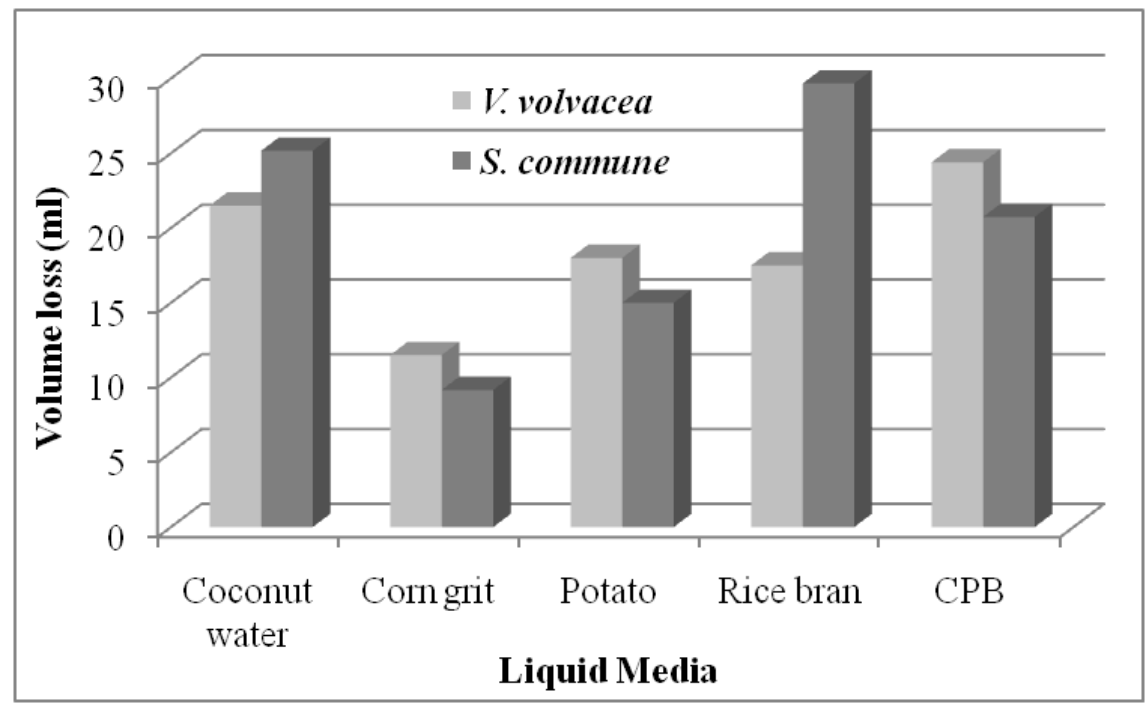

Fig. 2 - Volume loss of culture spent of $V$. volvacea and $S$. commune after 12 days of incubation. $\mathrm{CPB}$; commercial potato broth.

\section{Antioxidant activity}

Antioxidant is any substance that inhibits the damaging effects of free radicals brought by oxidation. Phenolic acids, flavonoids, carotenoids, tocopherol and ascorbic acids are the most common antioxidants contained in foods (Barros et al. 2008). The appropriate method to obtain the significant antioxidant compounds from medicinal mushrooms is through submerged cultivation (Elisashvili 2012). The DPPH radical scavenging activity of the liquid culture of mycelia of the two tested mushrooms was determined. Figure 3 shows the radical scavenging activities of $V$. volvacea and $S$. commune grown in different indigenous liquid media. Apparently, in both mushrooms, all liquid cultures possess radical scavenging activity against free radicals. Among the different 
indigenous liquid culture of $V$. volvacea, coconut water significantly recorded the highest activity of $21.19 \%$. This was followed by rice bran and potato broth which respectively recorded $18.21 \%$ and $12.95 \%$. However, the lowest activity was noted in corn grit with $12.08 \%$. In S. commune culture, on the other hand, the highest radical scavenging activity was registered in rice bran broth and coconut water respectively having means of $19.45 \%$ and $19.35 \%$. Although these values are found lower than the activity cathechin as control, this property observed in these liquid cultures of both mushrooms suggests a promising potentials in the pharmaceutical industries since antioxidant acts significant physiological functions in preventing free radical associated diseases.

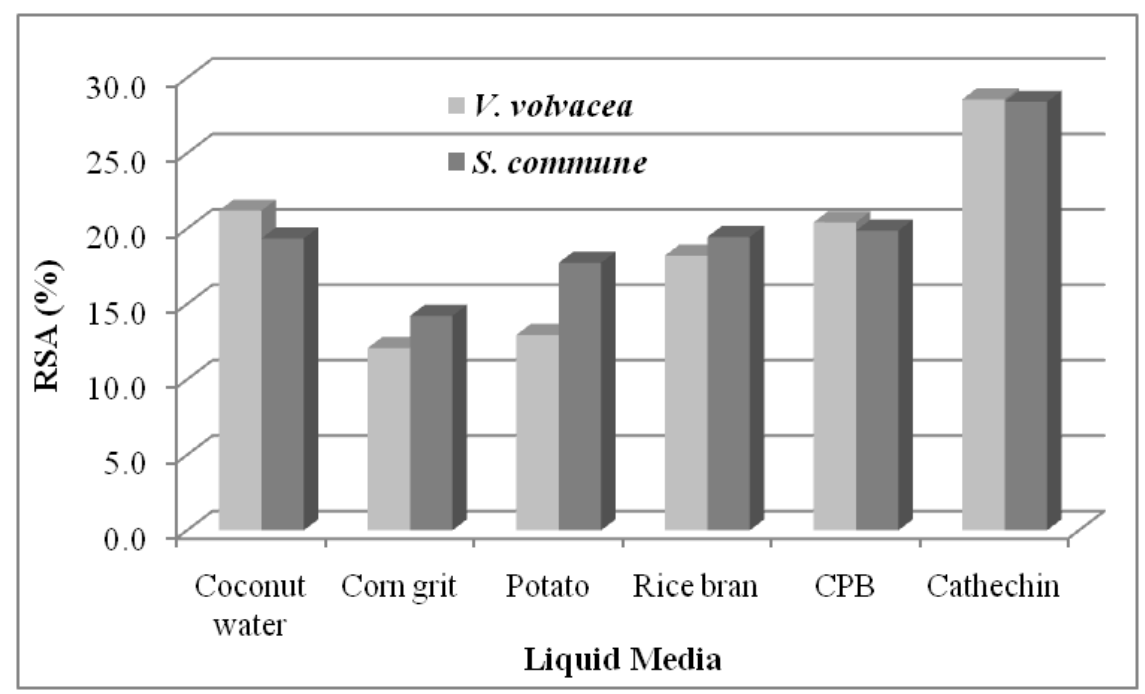

Fig. 3 - Radical scavenging activities of $V$. volvacea and $S$. commune in different indigenous liquid media. CPB; commercial potato broth.

\section{Total phenolic content}

One of the most important bioactive compounds with antioxidant activity is the total phenolic.Phenolic compounds exhibit antioxidant activity that are known to prevent heart ailments, fight cancer and even are anti-inflammatory agents (Doughari 2012). The total phenolic contents of the studied mushrooms cultured in indigenous liquid media were also determined in the present study and the results are presented in Figure 4. It can be seen that all media grown with $V$. volvacea mycelia exhibit phenolic contents. Rice bran broth significantly recorded the highest phenolic content of $23.19 \mathrm{mg}$ AAE/g sample while the corn grit showed the lowest content of $19.18 \mathrm{mg}$ AAE/g sample. On the other hand, $S$. commune grown in coconut water recorded the highest phenolic content of $25.52 \mathrm{mg}$ AAE/g sample, followed by rice bran broth with $25.18 \mathrm{mg}$ AAE/g sample. The lowest was registered in corn grit with a mean of $21.99 \mathrm{mg}$ AAE/g sample. Based on the data, the varying indigenous liquid media could affect the phenolic contents of $V$. volvacea and S. commune. This finding proved the previous work that the level of observed antioxidant activity depended on the bio-ecological differences of tested strains such as geographical origination, type of wood substrate, mycelial growth rate, and morphology, as well as the experimental conditions (Elisashvili 2012).

Several references are available on the positive correlation of total phenolic content in the mushroom extracts and their antioxidant properties. For instance, out of 23 mushroom species, Termitomyces heimii and Helvella crispa ranked as high-phenolics species and accordingly, by all assays $T$. heimii and T. mummiformis were found to be very high antioxidant varieties (Puttaraju et al. 2006). This observation is similar to that obtained in Ganoderma tsugae, Lentinus edodes, Volvariella volvacea, Lentinus tigrinus and Lentinus sajor-caju (Bimla \& Punita 2006, Cheung et al. 2003, Dulay et al. 2015b). In addition, total phenolic is the major naturally occurring antioxidant components found in Agrocybe cylindracea water extract, in the range of $23.74-30.16 \mathrm{mg} / \mathrm{g}$, which explains its high antioxidant properties. 


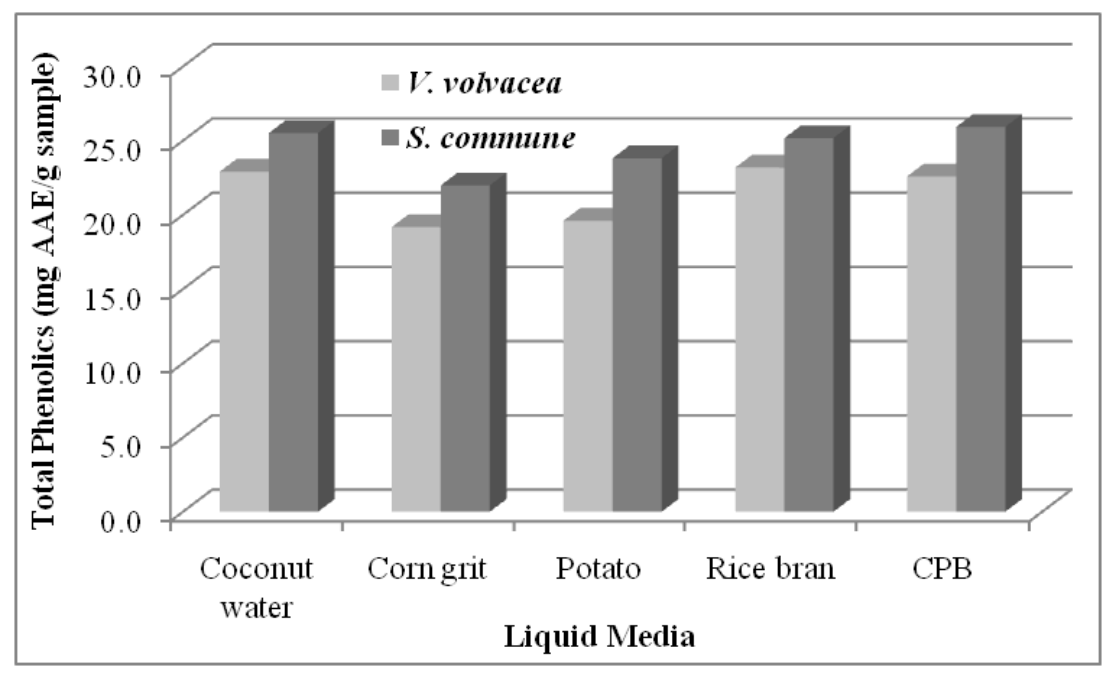

Fig. 4 - Total phenolic contents of $V$. volvacea and $S$. commune in different indigenous liquid media. CPB; commercial potato broth.

The antioxidant activity of mushroom may also vary in their fruiting body, mycelia and spent media. The total antioxidant components of $A$. cylindracea were 30.46, 27.72, and 24.57 $\mathrm{mg} / \mathrm{g}$ for fruiting bodies, mycelia, and filtrate, respectively (Tsai et al. 2006). The mycelia Pholiota alnicola, Lepista personata, Trametes versicolor, Volvariella bombycina, Schizophyllum commune, Suillus luteus, and Lentinus edodes showed more than 20\% antioxidant activity (Badalyan 2003). Evaluation of antioxidant properties of methanolic extracts from Grifola frondosa, Morchella esculenta, and Termitomyces albuminosus mycelia showed high antioxidant (85.4\%-94.7\%) at 25 $\mathrm{mg} / \mathrm{ml}$ (Mau et al. 2004).

In the current study, therefore, we evaluated the different indigenous liquid media for the mycelial production and antioxidant activity. It was found out that the different indigenous liquid media influenced the mycelia growth, volume loss, radical scavenging activity and total phenolic content of the two mushrooms. The total phenolic content in the mushrooms positively correlated on their antioxidant properties. This information on the antioxidant potential of these edible mushrooms would require further exploration for human health benefits.

\section{References}

Badalyan SM. 2003 - Edible and medicinal higher basidiomycetes mushrooms as a source of natural antioxidants. International Journal of Medicinal Mushrooms 5, 153-162.

Barros L, Falcão S, Baptista P, Freire C, Vilas-boas M, Ferreira ICFR. 2008 - Antioxidant activity of Agaricus sp. mushrooms by chemical, biochemical and electrochemical assays. Food Chemistry 111, 61-66.

Bimla N, Punita B. 2006 - Aluminium-induced imbalance in oxidant and antioxidant determinants in brain regions of female rats: protection by centrophenoxine. Toxicology Mechanisms and Methods 16, 21-25.

Bustillos RG, Dulay RMR, Bauto JJ, Pascual F, Baltazar K, Bunag HW, Macatula A, Nicolas MA, Torres MAM, Nillosa JC, Dela Cruz JC, Kalaw SP, Reyes RG. 2014 - Mycochemical profile of mycelia and fruiting bodies of Panaeolus cyanescens and its optimal submerged culture conditions for antioxidant properties. International Journal of Pure and Applied Bioscience 2(6), 175-181.

Chennupati S, Valluru S, Bhogavalli P. 2012 - Studies on antioxidant capacity of petroleum ether and acetone extracts of Pleurotus florida. Asian Journal of Biochemical and Pharmaceutical Research 2(2), 82-88. 
Cheung LM, Cheung PCK, Ooi VEC. 2003 - Antioxidant activity and total phenolics of edible mushroom extracts. Food Chemistry 81, 249-255.

Doughari J. 2012 - Phytochemicals: extraction methods, basic structures and mode of action as potential chemotherapeutic agents. In V. Rao (Ed.), Phytochemicals - A Global Perspective of Their Role in Nutrition and Health (pp. 1-33). Croatia: InTech.

Dulay RMR, Cabalar AC, De Roxas MJB, Concepcion JMP, Cruz NE, Esmeralda M, Jimenez N, Aguilar JC, De Guzman EJ, Santiago JQ, Samoy JR, Bustillos RG, Kalaw SP, Reyes RG 2015a - Proximate composition and antioxidant activity of Panaeolus antillarium, a wild coprophilous mushroom. Current Research in Environmental \& Applied Mycology 5(1), $52-59$

Dulay RMR, Flores KS, Tiniola RC, Marquez DHH, Dela Cruz AG, Kalaw SP, Reyes RG. $2015 b$. - Mycelial biomass production and antioxidant activity of Lentinus tigrinus and Lentinus sajor-caju in indigenous liquid culture. Mycosphere 6(6), 659-666

Eguchi F, Kalaw SP, Dulay RMR, Miyasawa N, Yoshimoto H, Seyama T, Reyes RG. 2015. Nutrient composition and functional activity of different stages in the fruiting body development of Philippine paddy straw mushroom, Volvariella volvacea (Bull.:Fr.) Sing. Advances in Environmental Biology 9(22), 54-65.

Elisashvili V. 2012 - Submerged cultivation of medicinal mushrooms: bioprocesses and products (review). International Journal of Medicinal Mushrooms 14(3), 211-239.

Jose N, Janardhanan K. 2000 - Antioxidant and antitumour activity of Pleurotus florida. Current Science 79(7), 941-943.

Ma Z, Ge L, Lee ASY, Yong JWH, Tan SN, Ong ES. 2008 - Simultaneous analysis of different classes of phytohormones in coconut (Cocos nucifera L.) water using high-performance liquid chromatography and liquid chromatography-tandem mass spectrometry after solidphase extraction.Analytica Chimica Acta 610, 274-281.

Mau J, Chang C, Huang S, Chen C. 2004 - Antioxidant properties of methanolic extracts from Grifola frondosa, Morchella esculenta and Termitomyces albuminosus mycelia. Food Chemistry 87, 111-118.

Menaga D, Ayyasamy P. 2012 - Effect of horse gram on the cultivation of Pleurotus florida mushroom and their phytochemical analysis and antimicrobial activity. International Journal of Research in Pharmaceutical Sciences 3(1), 140-145.

Nagendra PMN, Sanjay KR, Shrauya KM, Vismaya MN, Nanjunda SS. 2011 - Health benefits of rice bran. Nutrition and Food Science 2011, 1-3.

Preeti A, Pushpa S, Sakshi S, Jyoti A. 2012 - Antioxidant mushrooms: a review. International Research Journal of Pharmacy 3(6), 65-70.

Puttaraju GN, Venkateshaiah SU, Dharmesh SM, Nanjaraj Urs SM, Somasundaram RJ. 2006 Antioxidant activity of indigenous edible mushrooms.Agricultural Food Chemistry 54, 9764-9772.

Qui U, Lui Y. 2000 - Fruitbody production in basidiomycetes. Applied Microbiological and Biotechnology 54, 141-152.

Reis F, Baros L, Martins A, Ferreira I. 2011 - Chemical composition and nutritional value of the most widely appreciated cultivated mushrooms: an inter-species comparative study. Food Chemistry 128, 674-678.

Reyes RG, Graßl W, Rau U. 2009 - Coconut water as a novel culture medium for the biotechnological production of schizophyllan. Journal of Nature Studies 7, 1655-3179.

Santoso U, Kubo K, Ota T, Tadokoro T, Maekawa A. 1996 - Nutrient composition of kopyor coconuts (Cocos nucifera L.). Food Chemistry 57, 299-304.

Shimada K, Fujikawa K, Yahara K, Nakamura T. 1992 - Antioxidative properties of xanthan on the autoxidation of soybean oil in cyclodextrin emulsion. Journal of Agricultural and Food Chemistry 40, 945-948.

Silverio CM, Vilela LC, Guitateo FL, Hernandez NB. 1981 - Mushroom culture on enriched composted sawdust. NSTA Technology Journal 7(1), 4-13. 
Slinkard K, Singleton VL. 1977 - Total phenol analyses: automation and comparison with manual methods. American Journal of Enology and Viticulture 28, 49-55.

Tsai S, Huang S, Mau J. 2006 - Antioxidant properties of hot water extracts from Agrocybe cylindracea. Food Chemistry 98, 670-677.

Yong JWH, Ge L, Ng YF, Tan SN. 2009 - The chemical composition and biological properties of coconut (Cocos nucifera L.) water. Molecules 14, 5144-5164. 